Steffen Kailitz

\title{
Zur Typologisierung der Regierungsformen Eine Antwort auf Frank Deckers Replik
}

\section{Kurzfassung}

Frank Decker legte in Heft 2/09 eine Replik zu meinem empirisch ausgerichteten Beitrag zur Fraktionsgeschlossenheit in Heft 3/08 der ZPol vor. Die darin vorgetragene Kritik an meiner Typologisierung der Regierungsformen krankt an einer unzureichenden Kenntnis der möglichen Form der Typen- und Typologiebildung - vor allem der Charakteristika primär heuristisch gebildeter Typen und polythetischer Typologien. Die fundamentale Widersprüchlichkeit des Beitrags von Decker zeigt sich nicht zuletzt darin, dass Decker dogmatisch für eine Präsidentialismus-Parlamentarismus-Dichotomie und gegen die Bildung von Mischtypen (z. B. Semipräsidentialismus) eintritt, um anschließend eine Typologie vorzulegen, in der er selbst zwei Mischtypen - quasi-präsidentiell und präsidentiell-parlamentarisch - bildet. 


\section{Inhalt}

1. Einleitung 48

2. Grundlegende Möglichkeiten der Konstruktion von Typen und der Anlage einer Typologie

3. Deckers Kritik an meiner Typologiebildung und am Begriff des Semipräsidentialismus

4. Deckers Positionierung zur Bildung einer Typologie der Regierungsformen

\section{Einleitung}

In seiner Replik auf meine empirische Untersuchung zur Fraktionsgeschlossenheit in den Regierungsformen (Kailitz 2008a) kritisiert Frank Decker meine Form der Typologisierung der Regierungsformen und stellt zwei eigene Typologisierungsvorschläge vor (Decker 2009). Die Frage der Typologisierung konnte aufgrund der empirischen Ausrichtung im Rahmen meines Beitrags nur recht knapp abgehandelt werden. ${ }^{1}$ Daher nutze ich gerne die Gelegenheit, diese zu erläutern. Verwunderlich ist, dass Decker in seinem Beitrag nicht auf die grundlegenden Möglichkeiten der Bildung einer Typologie eingeht. Im ersten Teil sollen daher zunächst die möglichen Formen der Typen- und Typologiebildung erläutert werden (dazu u. a. Collier/Laporte/Seawright 2008; Elman 2005; Lauth 2009). Im zweiten Teil des Beitrags widerlege ich die gegen meine Form der Typologisierung vorgetragenen Einwände Deckers. Im dritten Teil bringe ich meine Kritikpunkte an Deckers - einander widersprechenden - Typologisierungsvorschläge vor.

\section{Grundlegende Möglichkeiten der Konstruktion von Typen und der Anlage einer Typologie}

Zunächst ist es notwendig, die Unterschiede zwischen einem heuristischen und einem empirisch gebildeten Typ zu erläutern. Ein rein heuristischer Typ wird gebildet, indem aus einem Konzept (z. B. dem der „separation of powers“) ein

1 Vielleicht lässt sich die Replik Deckers daher besser als eine Replik auf die Gesamtheit meiner Beiträge zur Parlamentarismus-Präsidentialismus-Debatte der letzten Jahre und vor allem auf meine Habilitationsschrift ansehen (u. a. Kailitz 2004, 2006, 2007, 2008b). 
Typ abgeleitet wird. Der rein heuristische Typ ist in Deutschland besser bekannt als Idealtyp (zur Typenbildung von Weber ausführlich Rogers 1969). Folgt man Max Weber und Robert Winch (Winch 1947: 68), dann haben rein heuristische Typen keine Entsprechung in der Wirklichkeit. Die Position von Max Weber lautete: „Je schärfer und eindeutiger konstruiert die Idealtypen sind: je weltfremder sie also, in diesem Sinne, sind, desto besser leisten sie ihren Dienst, terminologisch und klassifikatorisch sowohl wie heuristisch" (Weber 1984: 39). Ein bereits von Weber angeführtes Beispiel für einen Idealtyp ist der rein ökonomisch-rational denkende Mensch, der in der Wirklichkeit nicht existiert. Ein rein empirischer Typ resultiert dagegen ausschließlich aus empirischen Beobachtungen ohne vorherige Konzeptualisierung. Rein empirische Typen bilden Forscher auf der Grundlage von Cluster- oder Faktorenanalysen (dazu u. a. Sodeur 1974; Winch 1947). Idealtypen wie der „Homo oeconomicus“ des Rational-Choice-Ansatzes dienen gewöhnlich der Modellbildung, empirische Typen schlicht zur Klassifikation empirischer Beobachtungen.

Aufgrund der Typenbildung durch die Klassifikation der realen Phänomene ist bei einer rein empirischen Typenbildung ausgeschlossen, dass es Probleme mit der Zuordnung von Phänomenen zu Typen gibt. Sehr wohl können die gebildeten Typen aber logisch inkohärent sein, da die Bildung des Typs eben nicht logisch von einem Konzept ausgeht. Mit Giovanni Sartori, Paul Lazarsfeld, Allen Barton und wohl den meisten Sozialwissenschaftlern erscheint mir aus diesem Grund eine rein empirische Typenbildung ohne eine vorherige Konzeptbildung für sozialwissenschaftliche Studien wenig ergiebig (dazu u. a. Lazarsfeld/Barton 1965: 155; Sartori 1970: 1038). Wenn aber rein empirische Klassifikationen kein akzeptabler Ausweg aus dem Problem der eindeutigen Zuordnung von Fällen zu Typen sind, dann müssen wir die Lösung des Problems innerhalb der Grenzen von in erster Linie heuristisch gebildeten Typologien suchen. Bei der Konstruktion einer Typologie sollte die „Leiter der Abstraktion“ (Sartori 1970), aber nicht so weit emporgestiegen werden, dass die empirische Realität aus dem Blick gerät.

In den Sozialwissenschaften bilden wir daher in unseren Forschungen meist weder rein heuristische noch rein empirische Typen. Die meisten sozialwissenschaftlichen Typologien weisen sowohl Elemente der theoretischen Konzeptualisierung als auch einer Beobachtung von Variablenkonfigurationen in der realen Welt auf. Beobachtbare Phänomene entsprechen häufig einem Typ nur teilweise und weisen gleichzeitig Elemente eines anderen Typs auf. Typenbildungen können wir dabei auf einem Kontinuum zwischen Webers in der Realität nicht vorkommendem, rein konstruiertem Idealtyp und einem ohne vorherige 
Konzeptbildung angelegten, aus einer empirischen Klassifizierung resultierenden empirischen Typ abtragen. Auf diesem Kontinuum siedelt etwa Howard Beckers und John McKinneys „konstruierter Typ“ (Becker 1950; McKinney 1966), der auf Konstruktion beruht, aber im Unterschied zu Webers „Idealtyp“ in der Wirklichkeit vorkommen kann, noch sehr nahe am heuristischen Pol. Susann Kluges „empirisch begründeter Typ“ (Kluge 1999) findet sich schon weiter weg vom heuristischen Pol, aber zugleich immer noch deutlich näher am heuristischen als am empirischen Pol. In meinem Beitrag habe ich mit empirisch begründeten Typen gearbeitet. ${ }^{2}$ „Bei dieser Typenbildung w[e]rden sowohl die empirischen Korrelationen von Merkmalen berücksichtigt als auch die zentralen theoretischen Sinnzusammenhänge zwischen den Merkmalen, die das Verhältnis von Regierung und Parlament bestimmen“ (Kailitz 2008a: 294). Im Unterschied zu Webers Idealtypus sowie Beckers und McKinneys „konstruiertem Typ“ wird dabei also darauf abgehoben, dass bei der Bildung der Typologie auch die beobachtbare Wirklichkeit in den Blick genommen wird. In Abgrenzung zum Begriff „Idealtyp“ soll der Begriff „empirisch (also: durch Beobachtung der Wirklichkeit) begründeter Typ" verdeutlichen, dass es sich nicht um einen „rein gedanklich gebildeten Typen“ handelt. Der Anspruch ist also, dass „keine empiriefernen Konstrukte produziert werden sollen“ (Kluge 1999: 86). Im Unterschied zum Idealtyp handelt es sich beim „empirisch begründeten Typ“ um eine Konfiguration, die in der Wirklichkeit in aller Regel häufig vorkommt. Empirisch begründete (konstruierte) Typen sind aber keine empirischen Typen. ${ }^{3}$ Es müssen also nicht alle Phänomene auch alle Merkmale

2 In einem früheren Aufsatz habe ich mit Blick auf Parlamentarismus und Präsidentialismus noch in Anlehnung an Max Weber von Idealtypen gesprochen (Kailitz 2006). Vor allem wenn man mit Blick auf die theoretische Konzeptbildung des Präsidentialismus von den „Federalist Papers“ (Hamilton/Madison/Jay 1993) ausgeht, ist dies auch nicht falsch. Die Bezeichnung „empirisch begründeter Typ“ trägt aber besser der Tatsache Rechnung, dass Elemente der Beobachtung empirischer Konfigurationen in die Typenbildung einfließen. Das gilt mit Blick auf den Parlamentarismus dabei weit stärker als mit Blick auf den Präsidentialismus. So gibt es im Unterschied zum Präsidentialismus beim Parlamentarismus kein theoretisch durchdachtes Grundkonzept, das der Einführung der Regierungsform vorausging. Vielmehr wurde die empirische Praxis des Parlamentarismus erstmals bei Bagehot auf der Grundlage der Beobachtung der britischen Verfassungswirklichkeit zusammengefasst und systematisch dem Grundkonzept des Präsidentialismus gegenübergestellt (Bagehot 1971).

3 Die Begriffsbildung Kluges kann hier zu Missverständnissen führen und hat es im Falle Deckers getan. So heißt es bei Decker etwa an einer Stelle mit Verweis auf Abweichungen vom empirisch begründeten Grundtyp des Parlamentarismus: „Von einem ,empirisch begründeten" Kriterium zu sprechen, erscheint deshalb übertrieben“" (Decker 2009: 176). 
vollständig aufweisen, die den empirisch begründeten Grundtypen (z. B. des Präsidentialismus) auszeichnen.

Aus Typen können Typologien gebildet werden. ${ }^{4}$ Eine Typologie dient immer dazu, Objekte zu einem bestimmten Zweck zu gruppieren. Es gibt zwei grundlegende Punkte, die eine Typologie erfüllen muss: Die Phänomene, die einem Typ zugeordnet werden, müssen sich möglichst ähnlich sein (interne Homogenität) und die Phänomene, die verschiedenen Typen zugeordnet werden, müssen sich möglichst stark unterscheiden (externe Heterogenität). ${ }^{5}$ Bei diesen beiden Merkmalen geht es vor allem um die Trennschärfe der gebildeten Typen. Jedes Phänomen darf nur genau einem Typ zugeordnet werden können. Das ist dann gewährleistet, wenn die Typen auf der Grundlage nur eines Kriteriums eingeteilt werden, das entweder erfüllt ist oder nicht. Auf den ersten Blick - wenn wir von dem ungeklärten Status der „Sekundärmerkmale“ absehen - erscheint Steffanis Unterscheidung von Parlamentarismus und Präsidentialismus auf der Grundlage der Frage, ob die Regierung aus politischen Gründen durch die Parlamentsmehrheit abberufbar ist (Steffani 1979b), als ein Beispiel für eine solche Klassifikation. ${ }^{6}$

Zuordnungsprobleme treten in einer Typologie - wenn sie nicht rein empirisch mithilfe von Cluster- oder Faktorenanalysen gebildet wurde - in aller Regel aber auf, sobald der Forscher mehr als ein Merkmal zur Klassifikation nutzt. Zweitens können selbst bei nur einem Klassifikationskriterium Zuordnungsprobleme von beobachteten Phänomenen zu Typen dadurch auftauchen, dass in der Wirklichkeit manche Katzen grau und nicht schwarz oder weiß sind. Mit Blick auf das Vorhandensein manchen Merkmals sind dann graduelle Abstufungen zwischen einem klaren Ja und einem klaren Nein möglich.

Von grundlegender Bedeutung für meine Auseinandersetzung mit Decker ist die Unterscheidung in monothetische und polythetische Typologien. Bei monothetischen Typologien müssen alle Elemente die Anforderungen nach

4 Das Beispiel des Idealtypus des „rational denkenden Menschen“ zeigt aber, dass die Bildung von Idealtypen nicht zwangsläufig in eine Typologiebildung münden muss.

5 In der Politikwissenschaft ist die Propagierung dieser Prinzipien mit dem Namen Giovanni Sartori verbunden (Sartori 1970: 1041). Tatsächlich ist dies aber der gemeinsame Nenner der Literatur zu Typologien seit den 30er-Jahren. Vgl. u. a. Hempel/ Oppenheim 1936: 85; Sodeur 1974: 119; Bailey 1994: 1; Kluge 1999: 27).

6 Wenn nur ein Merkmal zur Unterteilung von Typen genutzt wird, um Phänomene zu klassifizieren, wird in der Methodenliteratur in der Regel von einer Klassifikation gesprochen (vgl. u. a. Lauth 2009; Lauth/Pickel/Pickel 2009: 44). Klassifikationen können aber durchaus mit guten Gründen als eindimensionale Typologien interpretiert werden (Collier/Laporte/Seawright 2008: 153), die im Kern den gleichen Zweck wie mehrdimensionale Typologien erfüllen. 
Eindeutigkeit, Ausschließlichkeit und Vollständigkeit erfüllen. Auch um monothetische Typologien zu bilden, können Forscher mehrere Merkmale benutzen. Alle Merkmale sind aber dann als notwendig anzusehen und keines darf nicht erfüllt sein, damit der Forscher ein beobachtetes Phänomen einem Typ zuordnen kann. Erst die gleichzeitige Erfüllung aller Merkmale ist bei monothetischen Typologien hinreichend, um ein Phänomen einem Typen zuzuordnen (u. a. Bailey 1973: 21).

Wenn wir bei einer monothetischen Unterscheidung aber mehrere Merkmale zur Klassifikation nutzen wollen, funktioniert dies häufig wegen der Widersprüchlichkeit von Merkmalsausprägungen nicht mehr. Wer diesen Weg wählt, muss sich daher damit begnügen, mit einem Kriterium oder allenfalls zwei Kriterien die Phänomene zu gruppieren. Das daraus folgende Dilemma der monothetischen Typologie brachte Steffani (1979b: 38) folgendermaßen auf den Punkt: ,Je größer bei der [Typologisierung] die Anzahl der definitionsnotwendigen Strukturkriterien gewählt wird, desto weniger politische Systeme werden den dadurch begründeten Strukturtypen entsprechen. Je geringer die Zahl der Kriterien, desto allgemeiner, differenzierungsärmer und möglicherweise nichtssagender wird der konstruierte Typ“.

Mit Steffanis Weg, zunächst nur ein Kriterium für die Typologisierung der Regierungsformen zu nutzen, entsteht also das Problem, dass die Kategorien möglicherweise zu allgemein und zu differenzierungsarm sind. Steffani hakte dieses Problem schlicht damit ab, dass er das ausgewählte Merkmal als ,zentrales Kriterium" bestimmte. Damit ist das Dilemma aber nicht aufgelöst, denn jeder Forscher der Welt wird bei der Anlage einer Typologie, die nur auf einem Kriterium beruht, das Merkmal auswählen, das ihm für den Typus am charakteristischsten erscheint. Auch Steffani entging nicht dem Problem, dass vor allem die interne Homogenität der Typen aufgrund der Orientierung an nur einem Kernkriterium leidet. So fallen bei ihm Staaten wie Belgien, Österreich, Frankreich (V. Republik) und Russland (vor der Autokratisierung unter Putin) sämtlich unter die Kategorie des Parlamentarismus, während die Schweiz zusammen mit den USA und den lateinamerikanischen Präsidialsystemen in die Kategorie des Präsidentialismus eingeordnet wird.

Nun kann man das Merkmal „Abberufbarkeit der Regierung“ pragmatisch zur empirischen Einordnung von politischen Systemen in die Regierungsformkategorien nutzen, aber eine Klassifikation nach dem Merkmal der Abberufbarkeit der Regierung ist eben nicht das Gleiche wie eine Typologie nach dem Verhältnis von Parlament und Regierung. Daher hat Steffani auch weitere Merkmale, die das Verhältnis von Regierung und Parlament mitbestimmen, 
nicht aus der Typenbildung verbannt. Für die Auseinandersetzung mit Decker ist also folgender Punkt in Erinnerung zu behalten: Zwar muss man nicht immer alle Unterscheidungsmerkmale für die Zuordnung von Phänomenen zu den Typen nutzen. Wenn ein Unterscheidungsmerkmal nicht zum Zweck der empirischen Klassifikation genutzt wird, bedeutet dies aber keineswegs, dass es damit kein Teil des Grundtyps mehr wäre. Im strengen Sinne ist Steffanis Typologie daher auch keine monothetische Typologie. So spricht Steffani mit Blick auf die Abberufbarkeit/Nicht-Abberufbarkeit des Regierungschefs nämlich nicht von einem alleinigen Kriterium, sondern von einem „Primärkriterium“. Durch die Nennung von „Sekundärkriterien“ bestreitet er also nicht, dass zur Charakteristik von Parlamentarismus und Präsidentialismus mehr als nur das Merkmal der Abberufbarkeit/Nicht-Abberufbarkeit des Regierungschefs gehört. Die „Sekundärkriterien“ (z. B. die Möglichkeit/Nicht-Möglichkeit der Parlamentsauflösung) müssen die Grundtypen des Parlamentarismus und Präsidentialismus nicht aufweisen, um dem Grundtyp zugeordnet zu werden. Damit handelt es sich bei der Typologie von Steffani auf den zweiten Blick aber um eine polythetische Typologie.

Polythetische Unterscheidungen müssen den Kriterien der Eindeutigkeit, Ausschließlichkeit und Vollständigkeit nicht im gleichen Sinne entsprechen wie monothetische Unterscheidungen. Die Anforderung an solche Typologien ist, dass sich die Phänomene in einer Kategorie mit Blick auf die Merkmale, nach denen typologisiert wird, stärker ähneln als die Phänomene in den anderen Kategorien. Die Phänomene in einer Kategorie müssen ,eine wesentliche Anzahl gemeinsamer Merkmale besitzen" und jedes der einen Typ bestimmenden Merkmale soll „,von einer größeren Zahl von Elementen besessen werden“ (Sodeur 1974: 21; dazu inhaltlich ungefähr genauso Bailey 1973: 21 und Kluge 1999: 79). Wenn nicht ein für die Zuordnung zu einem Typ notwendiges Kriterium oder mehrere Kriterien ausdrücklich benannt werden, ist kein Merkmal als notwendig anzusehen, um ein Phänomen einem Typ zuzuordnen (dazu auch Sokal/Peter H. Sneath 1963: 14). Während eine monothetische Unterscheidung nur die Aussage erlaubt, dass ein Phänomen zu einem Typ gehört, erlaubt eine polythetische Typologie eine graduelle Abstufung der Zugehörigkeit zu einem Typ. Bleiben wir der Einfachheit halber zunächst bei einer eindimensionalen Klassifikation der Regierungsformen anhand des Verhältnisses von Regierung und Parlament. Sind alle Merkmale des Grundtyps erfüllt, lässt sich auch von einem „reinen“ $\mathrm{Typ}^{7}$ sprechen. In dieser Hinsicht lassen sich die USA als Vertreter eines reinen Präsidentialismus präsentieren. In einem eindimensionalen

7 „Rein“ ist dabei wertneutral und nicht in einem normativen Sinne gemeint. 
Merkmalsraum lassen sich aber auch alle realen Phänomene zwischen den Grundtypen verorten. Unabhängig davon, ob man Zwischentypen bildet, lassen sich Abstufungen machen bei der Frage, ob ein Phänomen nur Merkmale eines Grundtyps aufweist oder ob sich die Merkmale der Grundtypen mischen (dazu u. a. Hempel 1972: 87). Mit Blick auf bestimmte Merkmalskombinationen können Mischtypen gebildet werden, sie müssen es aber nicht. Wenn sich die Merkmale mischen, lässt sich vielmehr auch festlegen, welche Merkmalskombination zur Zuordnung zu welchem Grundtyp führt.

In der Politikwissenschaft herrschte in den letzten beiden Jahrzehnten der polythetische Typologisierungsansatz vor. Typisch für eine solche Typologiebildung ist etwa Arend Lijpharts Unterscheidung in Mehrheits- und Konsensusdemokratien (1999). So gibt es in der beobachtbaren Wirklichkeit keine einzige Demokratie, die alle zehn Merkmale einer Konsensusdemokratie erfüllt. Auch der Gedanke einer dichotomischen Gegenüberstellung von Demokratie und Autokratie im Sinne von „bounded wholes“ (Sartori 1987: 184), bei denen alle Merkmale entweder präsent sind oder nicht, wurde von den meisten Forschern inzwischen zugunsten der Annahme eines Kontinuums von der Demokratie zur Autokratie aufgegeben. Das Grundproblem ist, dass bei einer dichotomen Gegenüberstellung im Grunde nur einer der Zustände als ,bounded whole“ definiert werden kann. Im Falle Sartoris ist dies die Demokratie. Bei der Nichterfüllung nur eines der Definitionsmerkmale wird dann das Regime als nicht-demokratisch klassifiziert. Damit wird das Gegenüber zur Restekategorie ohne eigenständige Merkmalsbestimmung. In der neueren politikwissenschaftlichen Forschung plädieren David Collier und andere (Collier/Levitsky 1997; Collier/ Adcock 1999) dafür, Typen, die nicht alle Merkmale eines Grundtyps vollständig aufweisen, als ,,verminderte Subtypen“ anzusehen. Dies ermöglicht es etwa bei einer dichotomen Gegenüberstellung, beide Konzepte zu definieren und je nach Merkmalsausprägungen die beobachtbaren Phänomene eher dem einen oder anderen Grundtyp zuzuordnen.

\section{Deckers Kritik an meiner Typologiebildung und am Begriff des Semipräsidentialismus}

Decker beansprucht, Klarheit in die Typologisierungsdebatte um Regierungsformen zu bringen, während Matthew Søberg Shugart und John Carey die Unterscheidungskriterien nur „,vernebeln“ und meine Ausführungen sogar 
„noch hinter [Shugart und Carey] zurückbleiben“ (2009: 176) würden. ${ }^{8}$ Konkret strebt Decker eine „Synthese der angelsächsischen und deutschen Typologisierungsbemühungen“ (Decker 2009: 173) an. Zugleich wandelt er aber erklärtermaßen in den Fußstapfen einer „deutschen Schule“ um Steffani, deren Vorgehen sich als „wesentlich überzeugender erwiesen habe“ (Decker 2009: 177). Nun gibt es so etwas wie eine voneinander klar abgrenzbare deutsche und angelsächsische Typologisierungsbemühung oder gar nationale „Schulen“ in der globalen Forschungswelt des 21. Jahrhunderts nicht. Decker möchte mit diesen hochtrabenden nationalen Etiketten anscheinend lediglich den Typologisierungsvorschlag von Steffani einerseits und die Typologisierungsbemühungen von Shugart, Carey und mir andererseits voneinander scheiden - obgleich ich in der Kategorisierung Deckers eigentlich der „deutschen“ Forschungswelt zugerechnet werden müsste. ${ }^{9}$

Von der Verfehltheit der Gegenüberstellung nationaler Schulen abgesehen, macht Decker eine falsche Front auf. Ich nehme in allen meinen Arbeiten zur Unterscheidung der Regierungsformen positiv Bezug auf Steffanis Arbeiten. ${ }^{10}$ Bei allem Respekt vor Steffanis Beiträgen zur Unterscheidung der Regierungsformen habe ich aber einen anderen Weg als er beschritten. Der Parlamentarismus ist ein Regierungssystem der Fusion von Regierung und Parlamentsmehrheit, der Präsidentialismus dagegen ein Regierungssystem der Unabhängigkeit

8 Die mit meinen Schriften angegriffenen Publikationen sind Shugart/Carey 1992; Shugart 1993, 2005.

9 Während Shugart und ich von Decker als „Gegner“ in der Kontroverse definiert werden, behauptet er mit Blick auf Alan Siaroff erstaunlicherweise, dass ,hartnäckige Festhalten an der klassischen Dichotomie" durch Steffani habe bei diesem Früchte getragen (2009: 183). Tatsächlich folgt Siaroff in dem von Decker angesprochenen Aufsatz aber keineswegs Steffani und unterscheidet lediglich zwischen zwei Regierungsformen. Er geht vielmehr wie auch Shugart und ich anhand des Kriteriums der Stärke des Präsidenten wegen der auch von mir und Shugart kritisierten Heterogenität der Semipräsidentialismuskategorie bei der Typologisierung noch über eine Dreiteilung der Regierungsformen hinaus: „In summary, then, as an alternative to the shortcomings of the concept of 'semipresidentialism', we may describe electoral democracies as one of: presidential systems, parliamentary systems with presidential dominance, parliamentary systems with a presidential corrective and parliamentary systems with figurehead presidents - plus, of course, parliamentary systems with figurehead monarchs" (Siaroff 2003: 309). Wie nahe die Ausführungen von Siaroff den kritisierten Ausführungen von mir stehen, mag besonders folgender Satz verdeutlichen: „The key initial distinction involves whether the head of state is also the head of government (a unified executive) as opposed to the head of state and government being separate (a dual executive)“ (Siaroff 2003: 295).

10 Konkret sind dies Steffani 1979a, 1983, 1992, 1995, 1996. 
von Regierung und Parlamentsmehrheit. Aus dieser Bestimmung lassen sich systematisch die Merkmale ableiten, die zu den Grundkonzepten Parlamentarismus und Präsidentialismus gehören.

Tabelle 1

Empirisch begründete Grundtypen von Parlamentarismus und Präsidentialismus

\begin{tabular}{ll}
\hline $\begin{array}{l}\text { Empirisch begründeter Grundtyp } \\
\text { des Parlamentarismus }\end{array}$ & $\begin{array}{l}\text { Empirisch begründeter Grundtyp } \\
\text { des Präsidentialismus }\end{array}$ \\
\hline 1. Das Parlament kann die & 1. Das Parlament kann die \\
Regierung(sspitze) abberufen. & Regierung(sspitze) nicht abberufen. \\
2. Der Premierminister kann dem & 2. Der Präsident kann dem Parlament \\
Parlament eine Vertrauensfrage stellen. & keine Vertrauensfrage stellen. \\
3. Die Parlamentswahl und die & 3. Die Parlamentswahl und die \\
Bestellung der Regierung sind & Bestellung der Regierung sind strikt \\
miteinander verflochten. Das Volk & voneinander getrennt. Das Volk wählt \\
wählt nur das Parlament (eingleisige & $\begin{array}{l}\text { Präsident und Parlament } \\
\text { (zweigleisige Legitimationsschiene). }\end{array}$ \\
Legitimationsschiene). & 4. Der Präsident kann das Parlament \\
4. Die Regierungsspitze kann das & nicht auflösen. \\
\hline
\end{tabular}

Eigene Zusammenstellung. Für eine nähere Begründung siehe Kailitz 2007.

Decker wendet gegen die Typenbildung ein, dass ich das Recht auf Parlamentsauflösung der Regierungsspitze als Teil des empirisch begründeten Grundtypus des Parlamentarismus aufführe, obgleich ich selbst in meinen Schriften nachgewiesen habe, dass in fast einem Viertel der heutigen präsidentiellen Demokratien der Präsident unter bestimmten Voraussetzungen das Parlament auflösen kann (Kailitz 2006: 40). Der Einwand geht jedoch an der Sache vorbei. Bei einem empirisch begründeten Grundtypus müssen - wie im ersten Teil erläutert - die beobachtbaren Phänomene nicht alle Merkmale aufweisen, die zum Grundtyp gehören, um diesem zugerechnet zu werden. Erst auf der Grundlage der Bestimmung eines heuristisch gebildeten Grundtyps erfolgt etwa meine von Decker sogar zitierte Aussage, dass diese präsidentiellen 
Demokratien damit „deutlich vom Idealtyp des Präsidentialismus“ (Kailitz 2006: 40) abrücken. ${ }^{11}$

Ich halte es für legitim und sinnvoll, nach der Bedeutung von Merkmalen zu unterscheiden, um eine zweifelsfreie empirische Zuordnung bei der Klassifikation von empirischen Phänomenen zu erreichen. Das kann man, wenn man zur Klassifikation schreitet, wie Steffani oder Decker auch durchaus eine Unterscheidung in Primärmerkmale und Sekundärmerkmale nennen (siehe Steffani 1962; Decker 2009). Es scheint Decker entgangen zu sein, dass auch ich, um bei der Klassifikation meiner Untersuchungsfälle Widersprüche zu vermeiden, nur die beiden mir am wichtigsten erscheinenden der vier Merkmale für die empirische Klassifikation genutzt habe, nämlich die Regelung von Auswahl (Direktwahl versus Bestimmung auf der Grundlage der Kräfteverhältnisse im Parlament) und Abberufbarkeit des Regierungschefs (Misstrauensvotum versus Nicht-Abberufbarkeit und Amtsenthebungsverfahren). ${ }^{12}$ Es handelt sich dabei genau um jene beiden Merkmale, die Decker erstaunlicherweise als vermeintlichen Gegenentwurf zu meiner Typologisierung in seiner Replik schließlich selbst zur Bildung seiner Typologie nutzt.

Decker übersieht bei seiner Kritik an meiner Bildung der empirisch begründeten Grundtypen, dass unabhängig davon, ob man ein Merkmal (z. B. Parlamentsauflösung) nur noch als „Sekundärmerkmal“ oder gar als „Tertiärmerkmal“" auffasst, alle Merkmale des Typs, die man aufführt, weiterhin Teil des Grundtyps sind. Es ist ein Trugschluss zu glauben, die Degradierung eines Merkmals zu einem „Sekundärmerkmal“ löse das Problem in Luft auf, dass nicht alle Phänomene entweder alle Merkmale des Grundtyps Parlamentarismus oder alle Merkmale des Grundtyps Präsidentialismus erfüllen. Die „Sekundärmerkmale“ Steffanis lassen sich aber lediglich ,zumeist oder vornehmlich“ (Steffani 1995: 636) einem der beiden Grundtypen Parlamentarismus

11 Wer im strengen Sinne mit Steffani davon ausgeht, dass zum Grundtyp des Parlamentarismus ein „unbeschränktes, jederzeit anwendbares Auflösungsrecht“" wie etwa in Großbritannien gehört, der kann auch zu dem Schluss kommen, dass weniger das gegenwärtige Viertel der präsidentiellen Demokratien mit einem Parlamentsauflösungsrecht als Kriseninstrument eine Abweichung vom Grundtyp des Präsidentialismus darstellt. Vielmehr sind dann die Fälle, in denen das Auflösungsrecht wie in der Bundesrepublik nur ein „Instrument der Krise, nicht der Opportunität“ (Steffani 1979b: 47) darstellt, durch die Nicht-Erfüllung des Kriteriums der selbstbestimmten Auflösung des Parlaments auf Veranlassung der Regierungsspitze vom Grundtyp des Parlamentarismus entfernt.

12 So hieß es in meinen Beitrag eindeutig: „Bei der empirischen Betrachtung wurden also Abweichungen vom empirisch begründeten Grundtypus bei zwei der vier Kriterien zugelassen. So gibt es etwa in Lettland nicht das Instrument der Vertrauensfrage und das Land wurde dennoch den parlamentarischen Demokratien zugeordnet" (2008a: 296). 
oder Präsidentialismus zuordnen. Bei einer genauen Betrachtung hat also auch Steffani eine polythetische Typologie vorgelegt und Decker streitet letztlich um des Kaisers Bart.

Ein weiterer Vorwurf Deckers lautet, dass ich nur eine „fehlerhafte Bestimmung des semi-präsidentiellen Grundtyps der ,dualen Exekutive“" (Decker 2009: 177) geleistet hätte. Die Systeme dualer Exekutive habe ich jedoch nicht primär über das Verhältnis von Parlament und Regierung bestimmt. Es heißt in meinem Text vielmehr: „Das Charakteristische eines semipräsidentiellen Systems, das treffender als System mit dualer Exekutive beschrieben werden kann, ist die Verteilung der Regierungsmacht auf zwei Ämter, Präsident und Premierminister." (2008a: 295) Anschließend habe ich dann die Merkmalsausprägungen des empirisch begründeten Grundtyps der dualen Exekutive mit Blick auf das Verhältnis von Parlament und Regierung bestimmt, um zu zeigen, dass diese Kriterien durchaus eine Abgrenzung des Systems der dualen Exekutive von den Grundtypen des Parlamentarismus und Präsidentialismus ermöglichen.

Auch bei dieser Kritik liegt das Problem darin, dass Decker die grundlegenden Möglichkeiten der Typenbildung anscheinend nur unzureichend kennt. In Anlehnung an Max Weber ist jede Theoriebildung ein „Versuch, Zusammenhänge durch eine theoretische Konstruktion auf wesentliche Bestandteile, Funktionsweisen und Charakteristika zu reduzieren" (Lauth 2004: 102). Bei der beschriebenen Verortung des Grundtyps einer dualen Exekutive mit Blick auf das Verhältnis von Regierung und Parlament habe ich zunächst die „Merkmale bzw. Vergleichsdimensionen, die der Typologie zugrunde gelegt werden sollen" (Kluge 2000), bestimmt.

Wer das Verhältnis von Regierung und Parlamentsmehrheit im Grundtyp einer dualen Exekutive in Abgrenzung zum parlamentarischen oder präsidentiellen Grundtyp konstruiert, kommt zu dem Schluss, dass in einem konsequenten System der dualen Exekutive das Recht zur Auflösung des Parlaments beim Premierminister liegen müsste, der ja auch vom Parlament abberufen werden kann. Das Recht des Parlaments, den Premierminister abzuberufen und das Recht des Premierministers, eine Parlamentsauflösung in die Wege zu leiten, gehören in einem theoretischen Sinnzusammenhang wie „Hand und Handschuh" (Loewenstein 1959: 217) zusammen. 
Typologisierung von Regierungsformen

Tabelle 2

Verhältnis von Parlament und Regierung im empirisch begründeten Grundtyp einer dualen Exekutive

\begin{tabular}{|c|c|}
\hline Regierung & $\begin{array}{l}\text { Verhältnis P } \\
\text { Präsident }\end{array}$ \\
\hline $\begin{array}{l}\text { Das Parlament kann die Regierung } \\
\text { bberufen. } \\
\text {. Der Premierminister kann dem }\end{array}$ & $\begin{array}{l}\text { 1. Das Parlament kann den Präsi- } \\
\text { denten nicht abberufen. } \\
\text { 2. Der Präsident kann dem Parlament }\end{array}$ \\
\hline $\begin{array}{l}\text { de stellen. } \\
\text { der in } \\
\text { st inschuss“" } \\
\text { irundla- }\end{array}$ & $\begin{array}{l}\text { llung } \\
\text { einander } \\
\text { völlig } \\
\text { erhält- } \\
\text { Wie in } \\
\text { gsform } \\
\text { ons- }\end{array}$ \\
\hline & icht auflösen. \\
\hline
\end{tabular}

Decker kommentiert meine Darlegung nun folgendermaßen: „Kaum entschuldigen lässt sich [...] die Formulierung des Kriteriums 4 bei Kailitz, wonach der Präsident das Parlament nicht auflösen kann, unterscheiden sich viele semi-präsidentielle Systeme doch gerade durch das Vorhandensein eines solchen Auflösungsrechts von ihren rein parlamentarischen Gegenstücken. Die prominentesten Beispiele sind Weimar und die V. Französische Republik" (Decker 2009: 177). Er glaubt also, mir einen schweren Fehler nachgewiesen zu haben, indem er zwei Beispiele nennt, die nicht alle acht Merkmale des Grundtyps der dualen Exekutive erfüllen. Bei der Konstruktion eines empirisch begründeten Typs geht es aber nicht darum, dass alle Fälle, die unter einen Typ gefasst werden sollen, auch alle Merkmale des Grundtyps aufweisen. Vielmehr geht man systematisch von einem theoretischen Sinnzusammenhang, konkret dem Verhältnis von Regierung und Parlament aus, ohne ein empiriefernes - also in der Wirklichkeit nicht vorkommendes - Konstrukt zu präsentieren.

Wenn in Tabelle 2 die Rede davon ist, dass der Präsident im empirisch begründeten Grundtypus der dualen Exekutive kein Auflösungsrecht hat, dann ist damit gemeint, dass bei einem dem empirischen Grundtyp entsprechenden Regime das Auflösungsrecht nicht im Ermessen des Präsidenten liegt. Es geht 
also nicht um die Frage, ob der Präsident rein formal das Parlament auflösen kann. Auch in nahezu allen parlamentarischen Demokratien hat das Recht zur Parlamentsauflösung nämlich formal nicht der Premierminister, sondern das Staatsoberhaupt (ob Präsident oder Monarch). Schweden ist seit der Verfassung von 1975 die Ausnahme (Kailitz 2004: 71). Beispielsweise in Großbritannien, so betonte etwa auch Steffani, ist „die Mitwirkung der Krone - zu deren Prärogative das Auflösungsrecht gehört - [...] praktisch ein formaler Akt" (Steffani 1979b: 46).

Bei der Betrachtung der Parlamentsauflösung durch den Präsidenten geht es weiterhin nicht um die Möglichkeit von Parlamentsauflösungen, die ein Staatsoberhaupt gewissermaßen als „Staatsnotar“ aufgrund eines in der Verfassung festgelegten Grundes vollziehen muss. So muss der Präsident in vielen Staaten ohne Handlungsspielraum wie in Albanien, Bulgarien oder Slowenien das Parlament auflösen, wenn eine Regierungsbildung misslingt. Es geht nur um jene Fälle, in denen das Recht zur Auflösung des Parlaments tatsächlich im Ermessen des Präsidenten liegt und er aufgrund eigener politischer Interessen aktiv werden kann. Genau auf die Existenz einer solchen Auflösungsmöglichkeit des Parlaments durch den Präsidenten aus politischen Gründen hebt auch Decker in den Fällen der Weimarer Republik und der V. Republik Frankreichs ab. Wer aber nun systematisch alle von mir in meinem Aufsatz behandelten Fälle mit dualer Exekutive daraufhin prüft, ob die Möglichkeit der Parlamentsauflösung im Ermessen des Präsidenten liegt, kommt zu folgendem Ergebnis: ${ }^{13}$

Tabelle 3

Parlamentsauflösungsrecht des Präsidenten

\section{Parlamentsauflösung liegt im Ermessen des Präsidenten}

Ja Nein

Finnland '45-'91, Finnland ab '91-2000; Griechenland ab '74; Irland;

Frankreich ab '59, Österreich ab '46; Island; Litauen ab '91; Bulgarien;

Portugal '76-'80; Mazedonien ab '91; Moldawien ab '91; Peru '80-'92;

Polen ab '89; Rumänien ab '90; Russland '91-2001;

Portugal ab '80; Slowakei ab '92; Slowenien ab '91;

Taiwan ab '92; Ukraine ab '91

Die Zusammenstellung basiert auf Shugart (2005: 336 f.) und einer ergänzenden eigenständigen Auswertung der Verfassungen der untersuchten Länder.

13 In meinem Text sage ich dabei klar, dass sich der empirisch begründete Grundtypus dualer Exekutive auf alle Systeme bezieht, in denen die Regierungsmacht auf Parlament und Präsident verteilt wird. Erst anschließend wird der Grundtyp der dualen Exekutive in die quasi-parlamentarischen Regime, die Systeme mit (wirklich) dualer Exekutive und quasi-präsidentielle Regime unterteilt. 
Es gab also im Untersuchungszeitraum unter meinen Fällen nur drei Länder mit dualer Exekutive mit der Konfiguration, die Decker als archetypisch für semipräsidentielle Systeme beschreibt. Gegenwärtig gibt es diese Konfiguration sogar nur noch in der V. Republik Frankreichs. Im Unterschied zu Deckers Behauptung sind also weder die V. Republik Frankreichs noch die Weimarer Republik als Archetypen der Demokratien mit dualer Exekutive anzusehen (siehe mit Blick auf Frankreich die klärenden Worte von Elgie 2009).

Vom theoretischen Sinnzusammenhang her betrachtet, müsste nun, um das entsprechende Kriterium des empirischen Grundtyps zu erfüllen, das Recht zur Parlamentsauflösung faktisch beim Premierminister und nicht beim Präsidenten liegen. Dazu sind einige erläuternde Worte notwendig, die im Rahmen eines empirischen Beitrags zur Fraktionsgeschlossenheit viel zu weit vom Thema geführt hätten. Tatsächlich sind in den Systemen mit dualer Exekutive in den Verfassungen häufig klare Regelungen dafür verankert, unter welchen Umständen das Parlament aufgelöst werden kann. Auch der Premierminister kann in diesen Fällen also nicht nach eigenem Ermessen handeln. Es lässt sich aber kaum bestreiten, dass in erster Linie die Verknüpfung zwischen erfolgreichem Misstrauensvotum und der Parlamentsauflösung, in zweiter Linie aber auch die Verknüpfung zwischen gescheiterten Regierungsbildungen und Parlamentsauflösungen Instrumente sind, die gewährleisten sollen, dass die Regierung mit dem Premierminister eine Parlamentsmehrheit hinter sich hat. Es handelt sich also eindeutig um Regelungen, bei denen es darum geht, eine Verflechtung von Regierung und Parlamentsmehrheit herzustellen. Diese Regelungen sind daher dem Grundtyp des Parlamentarismus zuzuordnen. In Staaten wie Finnland, Griechenland, Österreich, Irland und Moldawien liegt das Parlamentsauflösungsrecht auch „faktisch beim Regierungschef" (Patzelt 2006: 125) ${ }^{14}$ und nicht beim Staatsoberhaupt. ${ }^{15}$ In anderen Staaten mit dualer Exekutive wie Bulgarien, Litauen, Polen, Rumänien, Slowenien und der Slowakei folgt die Parlamentsauflösung automatisch einem erfolgreichen Misstrauensvotum gegen den Premierminister und/oder einer gescheiterten Regierungsbildung.

Auf der Grundlage der obigen Festlegungen des empirisch begründeten Grundtyps lässt sich also sagen, dass ein politisches System vom Grundtyp der dualen Exekutive abrückt, wenn das Recht der Parlamentsauflösung nicht nur

14 Die zitierte Aussage Patzelts ist dabei nur auf Finnland bezogen.

15 So heißt es etwa in der finnischen Verfassung: „On the basis of a reasoned initiative by the Prime Minister, the President may, after consulting the Speaker of Parliament and the various parliamentary factions, and at a time when Parliament is in session, dissolve Parliament by ordering that new elections be held" (Sektion 27 der 1991 modifizierten Verfassung Finnlands von 1919; inhaltlich gleich ist § 26 der Verfassung von 1999). 
formal, sondern auch faktisch in den Händen des Präsidenten und nicht des Premierministers liegt. Damit stellen die V. Republik Frankreichs und die Weimarer Republik Abweichungen vom empirisch begründeten Grundtyp dar. Durch die Verlagerung des Parlamentsauflösungsrechts in die Hände des Präsidenten in einem System dualer Exekutive entsteht nämlich ein Übergewicht zu dessen Gunsten. Deckers Anmerkung, ich würde durch eine „fehlerhafte Bestimmung des Auflösungskriteriums unfreiwillig“ darauf hinweisen, ,dass der Semi-Präsidentialismus in seinem präsidentiellen Zweig [...] von der Logik des Präsidentialismus in wichtigen Punkten“ (Decker 2009: 186 f.) abweiche, ist also verfehlt. Eine heuristisch (Idealtyp) oder vorwiegend heuristisch angelegte Konstruktion von Typen (empirisch begründete Typen) zielt nicht zuletzt darauf, Abweichungen zwischen den logischen Grundkonzepten und der Realität kenntlich zu machen. ${ }^{16}$

Laut der Überschrift von Deckers Replik steht die Verteidigung einer Parlamentarismus-Präsidentialismus-Dichotomie und mithin die Widerlegung der Notwendigkeit einer eigenständigen Kategorie für Systeme mit dualer Exekutive im Zentrum seiner Ausführungen. Dabei macht Decker wie bereits Steffani die Frage, ob der Semipräsidentialismus als ein eigenständiger Typ in der Regierungsformentypologie oder als ein Subtyp des Parlamentarismus verstanden wird, zu einer elementaren Frage der Typologiebildung. Damit wird dieser Frage ein übertriebenes Gewicht beigemessen. Je nach Fragestellung kann es sinnvoll sein, differenziertere oder weniger differenzierte Kategorien der Regierungsformen zu bilden. So habe ich in meinem Beitrag zur Fraktionsgeschlossenheit auch mit ,einem Präsidentialismus/Nicht-PräsidentialismusDummy gearbeitet, der die parlamentarischen und quasi-parlamentarischen Demokratien wie die Staaten mit dualer Exekutive zusammenfasst und sie den präsidentiellen und quasi-präsidentiellen Demokratien gegenüberstellt" (2008a: 297). Wenn es einen Zwang dazu gäbe, dass Politikwissenschaftler grundsätzlich nur zwei Typen von Regierungsformen bilden könnten, dann würde ich die Grenze entlang dieser Linie ziehen. ${ }^{17}$

16 Dieser Punkt wurde besonders stark von Max Weber mit Blick auf die Konstruktion von Idealtypen betont.

17 Unproblematisch sind solche Vergröberungen allerdings nie. Je weniger Kategorien wir bilden, desto undifferenzierter werden sie. Es treten also Probleme der internen Homogenität der Typen auf. Das Musterexemplar eines Systems der ,separation of powers“ (USA) wird bei dieser Grenzziehung etwa mit Regimen zusammengesperrt, die sich faktisch als Systeme der „concentration of powers“ (beim Präsidenten) bezeichnen lassen (z. B. Russland, Peru). 
Mit Blick auf meine Argumentation zu den Systemen mit dualer Exekutive meint Decker feststellen zu können, dass ich die Argumentation einer Mischung von Elementen des Grundtyps des Parlamentarismus und Präsidentialismus teile, obgleich ich sie an anderer Stelle mit Blick auf Duverger ausdrücklich kritisiert habe. Anscheinend hat Decker auch in diesem Punkt meine Argumentation nicht erfasst. So weichen viele reale präsidentielle Demokratien - wie bereits oben erläutert - etwa mit Blick auf ein Parlamentsauflösungsrecht des Präsidenten vom Grundtyp ab und ich rechne sie trotzdem zu den präsidentiellen Systemen.

Die Einführung eines dritten Grundtyps der dualen Exekutive begründe ich nicht mit einer Mischung von Merkmalen des Parlamentarismus und Präsidentialismus - wie Decker suggeriert - sondern damit, dass in den Systemen mit dualer Exekutive die Regierungsmacht nicht wie in Parlamentarismus und Präsidentialismus bei einem Organ liegt, sondern auf zwei Organe verteilt wird (Kailitz 2008a: 296).$^{18}$ Mit Blick auf dieses Kriterium erscheinen die Systeme mit dualer Exekutive als eine eigenständige Systemform, die gleichrangig neben Parlamentarismus und Präsidentialismus steht. Gegen diese - in meinen Text klar formulierte Position - hat Decker in seiner Replik kein einziges Argument angeführt. ${ }^{19}$

Um einige Punkte Deckers zu widerlegen, genügt ein schlichter Verweis auf meine tatsächlichen Ausführungen, die nicht mit der Wiedergabe durch Decker übereinstimmen. So wurde etwa der Terminus „duale Exekutive“ von mir nie so gebraucht, als ginge es lediglich um die ,übliche Trennung der Funktionen des Regierungschefs und Staatsoberhaupts“ (Decker 2009: 177). Der Begriff „duale Exekutive“ suggeriert auch keineswegs, dass damit nur eine Trennung zwischen den Ämtern des Regierungschefs und des Staatsoberhaupts gemeint sein könnte. Die Funktionen eines Staatsoberhaupts sind nämlich nur zeremonieller und eben nicht exekutiver Natur. Es kann auch keine Rede davon sein, dass ich mit der Wendung „duale Exekutive“ vom „üblichen politikwissenschaftlichen Sprachgebrauch“ (Decker 2009: 177) abweiche. Ich befinde mich vielmehr im Einklang mit dem Sprachgebrauch zahlreicher internationaler Kollegen (u. a. Blondel 1984; Elgie/McMenamin

18 Wenn es tatsächlich nur um die Mischung von Elementen des Parlamentarismus und Präsidentialismus ginge, würde ich Decker zustimmen, dass die Bildung von zwei Grundtypen ausreichend ist (siehe meine Ausführungen zu den polythetischen Typologien). Wir könnten dann nämlich je nach dem Grad der Mischung der Merkmale entscheiden, welchem Grundtyp wir das konkrete System zurechnen wollen.

$19 \mathrm{Zu}$ der Auseinandersetzung mit Deckers allgemeinen Gründen gegen die Bildung der Kategorie des „Semipräsidentalismus“ komme ich im vierten Teil. 
2006; Shugart 2005, 2003: 295 $)^{20}$ wenn ich von einer „dualen Exekutive“ dann spreche, wenn die Regierungsmacht auf zwei Ämter aufgeteilt wird (Kailitz 2008a: 295).

Warum belasse ich es nun in meinem Vorschlag für eine Regierungssystemtypologie nicht bei drei Kategorien, sondern fächere die Systeme mit dualer Exekutive in quasi-parlamentarische Systeme, Systeme mit wirklich dualer Exekutive und quasi-präsidentielle Systeme auf? Wenn wir etwa die Arbeiten von Robert Elgie $(1999,2004,1998)$ betrachten, der die Direktwahl des Präsidenten als Kriterium für die Klassifizierung nutzt, zeigt sich, dass Semipräsidentialismus ein Container ist, der keine ausreichende Homogenität der bezeichneten Phänomene aufweist. ${ }^{21}$ Das Kriterium der internen Homogenität einer Kategorie wird aber kaum befriedigend erfüllt, wenn so unterschiedliche gegenwärtige politische Systeme wie in Österreich, Frankreich und Russland in die gleiche Kategorie gepackt werden. Ich habe daher in meinem Beitrag vorgeschlagen, die Regime auf zwei Achsen zu bestimmen. Auf der für die Frage der Unterscheidung von Österreich, Frankreich und Russland entscheidenden Achse ordne ich dabei die Regierungsformen nach der Verteilung der Regierungsmacht. Es wird somit „Zwischen den unipolaren Exekutivtypen Parlamentarismus und Präsidentialismus einerseits, dem bipolaren Exekutivtyp andererseits“ unterschieden. „Auf dieser Achse ist je nach der Verteilung der Regierungsmacht zwischen tatsächlichen bipolaren Exekutiven und solchen zu unterscheiden, in denen ein Pol klar dominiert" (Kailitz 2008a: 296). Gerade aus der Aufspaltung des vagen Semipräsidentialismusbegriffs ergibt sich auch die Möglichkeit zu diskutieren, welche Systeme, die bislang etwa von Elgie als semipräsidentiell bezeichnet werden, sich sinnvoll dem Grundtyp des Parlamentarismus zuordnen lassen und welche nicht. So erscheint mir die Zusammenfassung der parlamentarischen und quasi-parlamentarischen Systeme

20 Weitere Forscher, die den Begriff des Semipräsidentialismus weiterverwenden, tun dies ausdrücklich nur deshalb, weil der Begriff eingeführt ist, obgleich sie den Begriff, duale Exekutive" für geeigneter halten (u. a. Linz 1994: 46, 82, Anm. 64).

21 Dies wird keineswegs nur von mir als Problem benannt. Robert Elgie leitet inzwischen etwa aus der zu großen Heterogenität der Kategorie folgende Forderung ab: „A very varied set of countries have semi-presidential constitutions. We should not use semipresidentialism as an explanatory variable. We should distinguish between different types of semi-presidentialism and explore the effects of each" (http://web.mac.com/relgie/The_Semi-presidential_One/Blog/Blog.html [Stand: 06.04.10]). 
in eine Kategorie durchaus sinnvoll, da beide Regimetypen in der Verfassungswirklichkeit keine bedeutsamen Unterschiede aufweisen. ${ }^{22}$

Meine Begriffsbildung „quasi-parlamentarisch“ als Bezeichnung für Demokratien, die zwar formal eine duale Exekutive haben, aber faktisch parlamentarisch funktionieren, kritisiert Decker mit einem Verweis darauf, wie Werner Kaltefleiter (1970: 71 f.) den Begriff „quasi-parlamentarisch“ benutzt und der Feststellung, dass meine Begriffsverwendung davon abweiche. Bei der Begriffsbildung beziehe ich mich aber nicht auf Kaltefleiter und verstehe unter dem Begriff auch etwas ganz anderes.

\section{Deckers Positionierung zur Bildung einer Typologie der Regierungs- formen}

Die Kategorie des Semipräsidentialismus bzw. der dualen Exekutive hat sich auf internationaler Ebene als eigenständige Regimekategorie auf ganzer Linie durchgesetzt. ${ }^{23}$ Um diesen Fast-Konsens aufzubrechen, sind sehr starke Argumente notwendig. Wie steht es aber nun um die Qualität der Argumente von Decker dafür, dass der Semipräsidentialismus unbedingt dem Grundtyp des Parlamentarismus zuzuordnen sei? Sein erstes Argument, dass Parlamentarismus und Semipräsidentialismus ,historisch im selben Entstehungszusammenhang gesehen werden" müssen, lässt sich nicht nachvollziehen. Was soll die Feststellung besagen, dass ,nach dem Umbruch in Osteuropa keines der dortigen Länder für die präsidentielle Regierungsform optiert hat" (Decker 2009: 183)? Will Decker damit etwa behaupten, dass die Regierungssysteme Russlands, der Ukraine und Weißrusslands alternativlos in die gleiche Kategorie wie die Regierungssysteme Großbritanniens und Deutschlands gepackt werden

22 Daher wurden bei den Analysen zur Fraktionsgeschlossenheit in meinem Beitrag diese beiden Typen auch zusammengefasst (2008a: 311).

23 Um keine ausufernde Literaturliste zu präsentieren, habe ich mich bei den folgenden Nennungen auf bedeutsame Beiträge der letzten Jahre beschränkt: u. a. Amorim Neto/ Costa Lobo 2009; Cheibub 2009; Cheibub/Chernykh 2009; Durst 2007; Elgie/Moestrup 2007, 2008; Elgie 2009; Kirschke 2007; Moestrup 2004; Protsyk 2005; Schleiter/MorganJones 2009; Shugart 2005; Tsai 2008. Wer das Semipräsidentialismuskonzept infrage stellt, plädiert dabei nicht wie Steffani für eine Beschränkung auf zwei Regierungsformkategorien, sondern für eine weitere Ausdifferenzierung der Betrachtung der Regierungsformen (Siaroff 2003; im Kern auch Shugart 2005 und Kailitz 2008b). 
müssen?24 Damit stünde er in der internationalen Gemeinschaft der Politikwissenschaftler nahezu allein, da es für eine solche Ansicht keine nachvollziehbaren Gründe gibt. ${ }^{25}$

In Asien oder Afrika finden sich ohnehin alle Regierungsformen. In den Regierungssystemdiskussionen Lateinamerikas, also einem Kontinent mit präsidentieller Prägung, ist zudem ausdrücklich der Semipräsidentialismus und nicht der Parlamentarismus der Bezugspunkt. ${ }^{26}$ Das zweite Argument Deckers lautet, dass „Systemwechsel sehr viel häufiger zwischen dem rein parlamentarischen und semi-präsidentiellen Typus“ stattfänden ,als zwischen einem von diesen und dem Präsidentialismus" (2009: 183). Regierungssystemwechsel sind generell selten. Interessant wäre es zu wissen, welche häufigen Wechsel zwischen rein parlamentarischen und semipräsidentiellen Regimen Decker beobachtet hat. Die deutsche (Wechsel vom Semipräsidentialismus der Weimarer Republik hin zum Parlamentarismus der Bundesrepublik) wie die französische Erfahrung (Wechsel vom Parlamentarismus der IV. Republik zum Semipräsidentialismus der V. Republik) verdeutlichen, dass es sich beim Wechsel vom Parlamentarismus zum Semipräsidentialismus gewöhnlich um einen grundlegenden Wechsel der Regierungssystemform handelt. Das Argument, dass es bei einem Wandel vom „semi-präsidentiellen zum rein parlamentarischen System“ keiner „,förmlichen Verfassungsänderung“ (Decker 2009: 183) bedürfe, ist zweitrangig. Im Gefolge von Steffani müsste es auch Decker um Änderungen der Verfassungspraxis und nicht des Verfassungstextes gehen. Tatsächlich

24 Steffani selbst ging auf diese Problematik auch nach 1990 kaum ein. In seinem Beitrag zu dem Band „Neue Regierungssysteme in Osteuropa und der GUS“ ordnet er die postkommunistischen Regierungssysteme in Zentral- und Osteuropa „fast alle“ (Steffani 1996: 56) - ohne konkrete Benennung von Ländern - dem Grundtyp des Parlamentarismus zu. Die Frage, ob die entstandenen Regierungssysteme sinnvoll unter den Begriff „Parlamentarismus“ gefasst werden können, erörterte Steffani nicht systematisch. Er wiederholte vielmehr lediglich Argumente, die er im Kern bereits seit 1962 vorgetragen hat (Steffani 1962), ohne ausführlicher auf die Veränderung der empirischen Lage, konkret auf die Verfassungslage in den postkommunistischen Staaten, einzugehen.

25 Das ist keineswegs nur meine Beobachtung. Um dies zu belegen, mag das folgende charakteristische Zitat zum Forschungsstand genügen: „Everyone seems to agree that the form of government in France, Portugal, and Ukraine is different both from the form of government in Italy, Germany, and Denmark on the one hand, and from the form of government in Brazil, the Philippines, and the United States on the other hand" (Cheibub 2009: 1395).

26 Dabei hat in Lateinamerika allerdings bislang einzig Brasilien kurzzeitig ein System mit wirklich dualer Exekutive eingeführt. Zu den Diskussionen vgl. u. a. Fernández 1987; Nogueira Alcalá 1986a; Nogueira Alcalá 1986b. Peru wies von 1980 bis 1992 ein quasipräsidentielles System auf. 
ist mir aber nicht einmal ein Fall bekannt, in dem ein Wechsel zwischen Parlamentarismus und Semipräsidentialismus ohne Verfassungsänderung stattgefunden hätte. Decker nennt auch kein einziges Beispiel für seine Behauptung, obgleich ihm das Argument so wichtig ist, dass er es in seinem Aufsatz sogar mehrfach anführt (etwa auch 2009: 185).

Deckers drittes Argument lautet, dass ,semi-präsidentielle und rein parlamentarische Systeme in ihrer grundlegenden Funktionsweise dahingehend überein[stimmen], dass am Ende die Parlamentsmehrheit über die Komposition und politische Ausrichtung der Regierung den Ausschlag" (Decker 2009: 183) gibt. Das trifft zu, bedeutet aber eben nicht - wie Decker behauptet -, dass die semipräsidentiellen Regime in ihrer ,grundlegenden Funktionsweise“ mit den parlamentarischen Regimen gänzlich übereinstimmen. Mit den präsidentiellen Systemen haben die Systeme dualer Exekutive nämlich gemeinsam, dass der Präsident nicht durch das Parlament bestimmt wird oder aus politischen Gründen abgewählt werden kann. Dadurch kann er seinen Teil der Regierungsmacht idealtypisch unabhängig von der Parlamentsmehrheit ausüben. Ein „divided government" ist mit Blick auf die Verteilung der Regierungsmacht nur in Präsidentialismus und Systemen dualer Exekutive möglich, nicht aber im Parlamentarismus. ${ }^{27}$ Der grundlegende Unterschied zwischen Parlamentarismus und Regimen mit dualer Exekutive sowie die grundlegende Gemeinsamkeit zwischen Regimen mit dualer Exekutive lässt sich aus der Sicht der PrinzipalAgent-Theorie folgendermaßen auf den Punkt bringen: In parlamentarischen Regimen gibt es nur einen volkslegitimierten Agenten (das Parlament), in Präsidentialismus und Systemen mit dualer Exekutive gibt es aber zwei (Parlament und Präsident; zum Forschungsstand ausführlicher Schleiter/MorganJones 2009: 874).

Unklar bleibt, warum Decker einerseits vehement gegen die Vorstellung eines Mischsystems zu Felde zieht, um dann in seiner Argumentation den nebulösen Begriff eines „,,unechten“ Mischsystems“ einzuführen, „das innerhalb der parlamentarischen Grundform zugleich Übereinstimmungen mit der präsidentiellen Regierungsweise aufweist" (Decker 2009: 193). Was gilt nun: Weist das Regierungssystem neben den Merkmalen des Parlamentarismus auch

27 Zur Problematik des „divided government“ Elgie 2001. Mit Blick auf starke Zweikammersysteme lässt sich die Wendung „divided government“ auch bei unterschiedlichen politischen Mehrheiten in beiden Kammern verwenden. Bei meinem Argument an dieser Stelle geht es aber ausschließlich um die Möglichkeit eines „divided government“, das durch die Verteilung der Regierungsmacht auf zwei Ämter entsteht. 
Merkmale des Präsidentialismus auf oder nicht? Wo liegt die Grenze zwischen einem ,echten“ und einem ,unechten“ Mischsystem?

Von der fehlenden Tragfähigkeit der Argumente Deckers gegen die Kategorie „Semipräsidentialismus“ abgesehen, gehen die Argumente alle an meinem Kernargument für die Eigenständigkeit von Systemen mit dualer Exekutive vorbei: In Systemen mit dualer Exekutive wird die Regierungsmacht im Unterschied zu parlamentarischen und präsidentiellen Regimen auf zwei Ämter verteilt.

Decker präsentiert selbst eine in der Typologie von Steffani wurzelnde Typologievariante, die er allerdings im Kern bereits durch die Vorstellung einer zweiten Typologie verwirft. Tatsächlich macht die an Steffani angelehnte Typologie keinen durchdachten Eindruck und kann daher wohl kaum als überzeugendes Argument für eine rein dichotome Klassifikation der Regierungsformen herhalten.

Bei der Bestimmung des Primärmerkmals (Abberufbarkeit bzw. NichtAbberufbarkeit der Regierung) folgt Decker schlicht Steffani. Für eine trennscharfe Abgrenzung der Regierungsformen ist allerdings selbst das von Decker hochgehaltene Steffani-Kriterium nicht über jeden Zweifel erhaben. Meine Analyse von 89 Demokratien von 1945-2004 hat ergeben, dass in der beobachtbaren Wirklichkeit die Grenzen zwischen einer Abberufbarkeit der Regierungsspitze aus politischen Gründen und einer Nicht-Abberufbarkeit der Regierungsspitze aus politischen Gründen bereits in den Verfassungstexten und noch weit stärker in der Verfassungswirklichkeit fließend sind (Kailitz 2004: 55-68, 81-92). So kann etwa gemäß der argentinischen (Art. 53 der Verfassung von 1994) und paraguayanischen Verfassung (Art. 225 der Verfassung von 1992) ein Amtsenthebungsverfahren auch wegen schlechter Amtsführung des Präsidenten eingeleitet werden. Diese Begründung ermöglicht einen Sturz wegen einer Veränderung der Mehrheitsverhältnisse im Parlament aus politischen Gründen. Oppositionsparteien dürften nämlich durchaus häufig der Ansicht sein, dass der Präsident der Regierungspartei sein Amt schlecht führt. Nur die höhere Hürde der notwendigen Mehrheit von zwei Dritteln der Parlamentarier, die einer Abberufung zustimmen müssen, unterscheidet damit die Regelung im Kern vom Misstrauensvotum in parlamentarischen Demokratien. Ohne auf diese Ergebnisse meiner Studie von 2004 konkret Bezug zu nehmen, räumt Decker die geschilderte Problematik in seiner Replik auch ein (2009: 195).

Ausdrücklich habe ich in meinem Beitrag, auf den Decker antwortet, vermerkt, dass für empirische Analysen - trotz dieser Problematik - die Fixierung auf Steffanis Kernkriterium der Abberufbarkeit der Regierung ,bestens geeignet 
[ist], weil alle Staaten klar zugeordnet werden können“" (Kailitz 2008a: 294). Allerdings müssen bei der „Bildung der Grundtypen [...] auch die empirischen und theoretischen Zusammenhänge mit den [...] anderen [zum Typus gehörigen] Merkmalen berücksichtigt werden“" (Kailitz 2008a: 294). Der Grund ist schlicht, dass die Konzepte von Parlamentarismus und Präsidentialismus mehr umfassen als das Kriterium der Abberufbarkeit der Regierung aus politischen Gründen bzw. der Nicht-Abberufbarkeit aus politischen Gründen. Das kann nicht ernsthaft bestritten werden und es wurde zumindest von Steffani auch nie bestritten. Er machte lediglich einen Kunstgriff, um bei der empirischen Klassifikation von Phänomenen Widersprüche zu vermeiden. Dazu erklärte er ein Merkmal zum ,zentralen Merkmal“, die übrigen Merkmale von Parlamentarismus und Präsidentialismus dagegen zu „Sekundärmerkmalen“. Ein grundlegendes Problem ist nun, dass Decker wie bereits Steffani die Bedeutung der „sekundären“ und „tertiären“ Merkmale im Rahmen seiner Typologie nicht erläutert.

Bei Deckers Benennung von „sekundären“ und „tertiären“ Merkmalen wird es zudem chaotisch. Es handelt sich bei dieser Variante der Typologie von Steffani daher um eine Verschlechterung im Vergleich zum Original. Bei den sekundären Merkmalen der Regierungsform taucht beim Präsidentialismus als erstes die „Volkswahl der Regierung“ auf, ein Pendant beim Parlamentarismus fehlt ebenso wie beim Kriterium eines „Bestätigungs- und Entlassungsrechts der Legislative“. Hinsichtlich der sekundären Merkmale zur „Struktur von Exekutive und Legislative" findet sich zum etwas nebulösen Kriterium der „Gegenzeichnung“ beim Parlamentarismus wiederum kein Pendant in der Merkmalsliste des Präsidentialismus. Bei den tertiären Merkmalen stellt Decker dem Einheitsstaat im Parlamentarismus die direkte Demokratie im Präsidentialismus gegenüber (Decker 2009: 189). Schon bei den „sekundären Merkmalen" vermengt Decker weiterhin Elemente der Regierungsformen mit ihren Konsequenzen (strenge Fraktions- und Koalitionsdisziplin versus geringe Fraktions- und Koalitionsdisziplin). Gar nicht nachvollziehbar ist es, andere Strukturmerkmale von Demokratien wie die Gegenüberstellungen von Einkammersystemen und Zweikammersystemen oder Einheitsstaaten und Zentralstaaten kurzerhand zu „,sekundären“ oder „tertiären“ Merkmalen von Parlamentarismus und Präsidentialismus zu erklären.

Die Unzulänglichkeit des Typologisierungsvorschlag von Decker zeigt sich nicht zuletzt an den Staaten, die er selbst weder dem Parlamentarismus noch dem Präsidentialismus zuordnen kann und die er mit dem Etikett „Superpräsidentialismus“" versieht. Damit sprengt er nämlich die von ihm propagierte 
dichotomische Unterscheidung in Parlamentarismus und Präsidentialismus. So wird etwa Russland ein „Systemwechsel hin zum Superpräsidentialismus“ (Decker 2009: 186) attestiert. ${ }^{28}$

Mit großem Eifer tritt Decker dafür ein, dass die Grenzen einer Parlamentarismus-Präsidentialismus-Dichotomie nicht überschritten und keine Mischtypen wie „Semipräsidentialismus“ gebildet werden. Angesichts dessen erscheint es widersinnig, dass er in seinem Beitrag dann selbst eine zweite Typologie entwirft, die mit den von ihm verfemten Mischtypen arbeitet. Sie unterscheidet zwischen vier und eben nicht dichotom zwischen zwei Regierungsformen. Deckers Aussage, dass sich „Steffanis dichotomische Unterscheidung [...] mühelos in das von Arend Lijphart 1984 erstmals vorgelegte vierteilige Typenschema" (Decker 2009: 199) integrieren lasse, ist unsinnig. Entweder man bildet zwei oder vier Typen von Regierungsformen; entweder man bildet Mischtypen oder man lehnt sie ab.

Decker präsentiert in seiner Replik eine Typologie, die fast vollständig der von Shugart (2005: 326) propagierten entspricht, also einem Typologieprodukt der aus Deckers Sicht eigentlich „vernebelnden“ angelsächsischen „Schule“. Ihm kann das auch auf keinen Fall entgangen sein, da er im Anschluss an die Darlegung der Typologie eine Aussage aus dem Text präsentiert, in dem Shugart die Typologie vorstellt. Paradoxerweise wird zusammen mit meiner Typologie im ersten Teil des Beitrags sogar just jene Typologie von Shugart kritisiert und korrekt zitiert, die Decker dann im zweiten Teil des Beitrags leicht variiert als eigenen Vorschlag unterbreitet (2009: 176). Die Länderbeispiele hat Decker allerdings nicht von Shugart übernommen. Im Fall der Schweiz trifft die $\mathrm{Zu}$ ordnung auch nicht so recht zu. In der Schweiz bestellt das Parlament nicht die Regierung, wie Decker (2009: 197) behauptet, sondern die Verteilung der Regierungsposten ist aufgrund der „Zauberformel“ festgelegt. Die Einordnung der V. Republik Frankreichs greift zudem zu kurz, da zwar der Premierminister, nicht aber der Präsident, der mit exekutiven Befugnissen ausgestattet ist, durch das Parlament bestellt wird.

Wie unausgegoren und widersprüchlich die Kritik Deckers an meinem Beitrag sowie seine eigene Positionierung in der Typologisierungsdebatte sind,

28 In Deckers zweitem Typologisierungsvorschlag (2009: 197; siehe aber etwa bereits Shugart 2005: 326), dessen Verhältnis zu seinem ersten Typologisierungsvorschlag gänzlich ungeklärt bleibt, ist dann allerdings keine Rede mehr von den „,superpräsidentiellen" Systemen. Der Grund ist letztlich, dass die Abgrenzung dieses Typus weder optimal über die Bestellung noch die Abberufbarkeit der Regierung klassifiziert werden kann. Vielmehr ist hier die in meinem Beitrag zur Klassifikation genutzte Frage der Machtverteilung in der Exekutive zu beachten. 
zeigt sich sowohl in der Grundanlage seines Beitrags als auch in den Details. Der Kern von Deckers Kritik zielt gegen die Bildung von Mischformen zwischen Parlamentarismus und Präsidentialismus, nur einige Seiten später bildet Decker dann selbst Mischformen, die er - unfreiwillig komisch - als „echte Mischsysteme“ (2009: 193) tituliert. Die äußerst widersprüchliche Argumentation geht bis in die Details. So heißt es bei Decker zunächst (2009: 180): „Die darauf fußende Typologie [Steffanis] hat den Vorzug, dass sie die Existenz von Mischsystemen logisch ausschließt. Selbst ein System wie das schweizerische, dessen parlamentarische oder präsidentielle Qualität sich dem Beobachter nicht unmittelbar erschließt, kann mit ihrer Hilfe eindeutig zugewiesen werden: Es gehört zum präsidentiellen Typus". Ich werde dann an dieser Stelle ausdrücklich dafür kritisiert, dass ich - im Einklang mit zahlreichen anderen Demokratieforschern - die Schweiz als „weder parlamentarisch noch präsidentiell“ (Kailitz 2006: 37) bezeichnet habe (Decker 2009: 180). Bei der Bildung seiner Typologie aufgrund der Merkmale Bestellung und Abberufung des Regierungschefs heißt es dann aber: „Bezieht man die Kreation der Regierung als Kriterium mit ein, so wird auch Steffanis Zuordnung der Schweiz zum präsidentiellen Typus fraglich. Die Schweizer Regierung, der siebenköpfige Bundesrat, ist zwar vom Parlament nicht abberufbar; sie muss jedoch von diesem in einer förmlichen Wahl bestellt werden. [...] Als Typus bewegt sich das Schweizer System so gesehen zwischen allen Stühlen. Es ist weder parlamentarisch noch präsidentiell“" (Decker 2009: 196).

Mit Blick auf die beiden in meinem Beitrag zur empirischen Klassifikation verwendeten Merkmale der Abberufbarkeit und der Bestellung der Exekutive ${ }^{29}$ lässt sich die Schweiz in der Tat nicht klar zuordnen. Decker schließt dabei aber fälschlich aus meiner Aussage, die Schweiz sei weder parlamentarisch noch präsidentiell, dass ich gegen die Grundanforderung an die Typologiebildung verstoße, nach der jedes Phänomen auch zugeordnet werden kann. Mit meiner Typologisierung kann die Schweiz aber durchaus eindeutig zugeordnet werden. Wenn man meine vier Kriterien auf die Schweiz anwendet, ist sie nach drei von vier Merkmalen präsidentiell: Es gibt kein Misstrauensvotum, keine Möglichkeit der Vertrauensfrage und kein Parlamentsauflösungsrecht der Regierung. Einzig das Fehlen einer dualen Legitimationskette von Regierung und Parlament deutet demnach in Richtung eines parlamentarischen Systems. Die Schweiz lässt sich mit meiner Typologie also klar einer Kategorie zuordnen. Meine Typologie ist in dieser Hinsicht ebenso vollständig wie die von Steffani. Das Ergebnis der Klassifikation greift aber zu kurz. Die Schweiz passt nicht so

29 Diese nutzt Decker dann im zweiten Teil seiner Replik analog zu mir. 
recht in die Kategorie des Präsidentialismus und an diesem mit Blick auf die Typologiebildung etwas unbefriedigenden Eingeständnis kommt auch Decker nicht vorbei.

\section{Zusammenfassung}

Das was an Deckers Argumenten durchaus richtig und wichtig ist, hat Steffani bereits vor mehreren Jahrzehnten vorgetragen. Ansonsten fällt der Beitrag Deckers deutlich hinter die originellen Gedanken Steffanis zurück. So bleibt Decker etwa mit Blick auf die „superpräsidentiellen“ Systeme ratlos, Steffani hat diese weiterhin als parlamentarisch angesehen. Steffani lässt sich daher zwar eine kategoriale Überdehnung des Konzepts Parlamentarismus vorwerfen, aber zumindest hat er im Unterschied zu Decker eine klare Position bezogen und sich nicht in Widersprüche verwickelt. Geradezu absurd wird die vehemente Argumentation gegen die Verwendung von Mischsystemen, wenn Decker am Ende eine eigene Typologie mit zwei Mischsystemen, den quasi-präsidentiellen (z. B. Schweiz) und den präsidentiell-parlamentarischen (z. B. Israel 19962001), vorlegt. Seine im Kern von Shugart übernommene Typologie steht im klaren Widerspruch zu dem titelgebenden Plädoyer von Decker.

Deckers Schlussbemerkungen in der Replik verdichten den Eindruck einer fundamentalen Inkonsequenz des Beitrags. Zunächst formuliert er selbst einen Vorschlag jenseits einer dichotomischen Parlamentarismus-PräsidentialismusUnterscheidung. Anschließend wendet er sich aber erneut scharf gegen die Bildung von Mischsystemen. Decker scheint tatsächlich nicht zu bemerken, dass er sich mit der Bildung seiner Mischsysteme jenseits der von Steffani propagierten klaren dichotomen Parlamentarismus-Präsidentialismus-Unterscheidung befindet. Dabei genügt es, einfach die Zahl der Regierungsformkategorien zu zählen, die er gebildet hat. Es ist nun ebenso legitim, eine dichotome wie eine drei- oder fünfstufige Typologie vorzulegen. Es ist auch ebenso legitim, eine monothetische wie eine polythetische Typologie zu entwickeln. Man sollte aber am Ende zumindest selbst wissen, was für eine Typologie man propagiert. 


\section{Literatur}

Amorim Neto, Octavio/Costa Lobo, Marina, 2009: Portugal Semi-Presidentialism (Re)considered. An Assessment of the President's Role in the Policy Process, 1976-2006, in: European Journal of Political Research 48, 234-255.

Bagehot, Walter, 1971: Die englische Verfassung, hrsg. und eingeleitet von Klaus Streufthau, Neuwied/Berlin (ursprgl. 1867).

Bailey, Kenneth D., 1973: Monothetic and Polythetic Typologies and Their Relationship to Conceptualization, Measurement and Scaling, in: American Sociological Review 38, 18-33.

Bailey, Kenneth D., 1994: Typologies and Taxonomies. An Introduction to Classification Techniques, Thousand Oaks.

Becker, Howard, 1950: Through Values to Social Interpretation: Essays on Social Contexts, Actions, Types, and Prospects, Durham.

Blondel, Jean 1984: Dual Leadership in the Contemporary World. A Step Toward Executive and Regime Stability?, in: Peel Kavanagh (Hrsg.), Comparative Government and Politics. Essays in Honour of Samuel E. Finer, London, 73-91.

Cheibub, José Antonio, 2009: Making Presidential and Semi-presidential Constitutions Work, in: Texas Law Review 88, 1375-1406.

Cheibub, José Antonio/Chernykh, Svitlana, 2009: Are Semi-presidential Constitutions Bad for Democratic Performance?, in: Constitutional Political Economy 20, 202-229.

Collier, David/Adcock, Robert, 1999: Democracy and Dichotomies: A Pragmatic Approach to Choices about Concepts, in: Annual Review of Political Science 2, 537-565.

Collier, David/Laporte, Jody/Seawright, Jason 2008: Typologies: Forming Concepts and Creating Categorical Variables, in: Janet M. Box-Steffensmeier/Henry E. Brady/David Collier (Hrsg.), The Oxford Handbook of Political Methodology, Oxford/New York, 152-173.

Collier, David/Levitsky, Steven, 1997: Democracy with Adjectives. Conceptual Innovation in Comparative Research, in: World Politics 49, 430-451.

Decker, Frank, 2009: Ist die Parlamentarismus-Präsidentialismus-Dichotomie überholt? Zugleich eine Replik auf Steffen Kailitz, in: ZPol 19, 169-203.

Durst, David C., 2007: The Perils of Semi-Presidentialism, in: The Review of Politics 69, 142-146.

Elgie, Robert, 1998: The Classification of Democratic Regime Types: Conceptual Ambiguity and Contestable Assumptions, in: European Journal of Political Research 33, 219-238. 
Elgie, Robert 1999: The Politics of Semi-Presidentialism, in: Robert Elgie (Hrsg.), Semi-Presidentialism in Europe, Oxford, 1-21.

Elgie, Robert, 2001: Divided Government in Comparative Perspective, Oxford/ New York.

Elgie, Robert, 2004: Semi-Presidentialism: Concepts, Consequences and Contesting Explanations, Dublin.

Elgie, Robert, 2009: Duverger, Semi-presidentialism and the Supposed French Archetype, in: West European Politics 32, 248-267.

Elgie, Robert/McMenamin, Iain, 2006: Divided Executives and Democratisation, Dublin.

Elgie, Robert/Moestrup, Sophia, 2007: Semi-presidentialism Outside Europe. A Comparative Study, London.

Elgie, Robert/Moestrup, Sophia, 2008: Semi-presidentialism in Central and Eastern Europe, Manchester.

Elman, Colin, 2005: Explanatory Typologies in Qualitative Studies of International Politics, in: International Organization 59, 293-326.

Fernández, Mario, 1987: Presidencialismo, parlamentarismo, y semipresidencialismo. Tres tesis sobre un sistema de gobierno para Chile, in: Cuadernos del Claeh 43, 77-98.

Hamilton, Alexander/Madison, James/Jay, John, 1993: Die Federalist Papers, übersetzt, eingeleitet und mit Anmerkungen versehen von Barbara Zehnpfennig, Darmstadt.

Hempel, Carl/Oppenheim, Robert, 1936: Der Typusbegriff im Lichte der Neueren Logik, Leiden.

Hempel, Carl G., 1972: Typologische Methoden in den Sozialwissenschaften, in: Ernst Topitsch (Hrsg.), Logik der Sozialwissenschaften, Meisenheim, 85-103.

Kailitz, Steffen, 2004: Parlamentarische, semipräsidentielle und präsidentielle Demokratien. Strukturen und Konsequenzen, Chemnitz.

Kailitz, Steffen, 2006: Parlamentarische, semipräsidentielle und präsidentielle Demokratien - idealtypische und reale Unterschiede der politischen Strukturen und Prozesse, in: Jahrbuch Extremismus \& Demokratie 18, 34-56.

Kailitz, Steffen, 2007: Der stille Abschied von der „seperation of powers“. Über die „Parlamentarisierung“ präsidentieller Demokratien, in: Sabine Kropp/ Hans-Joachim Lauth (Hrsg.), Gewaltenteilung und Demokratie. Konzepte und Probleme der „Horizontal Accountability“ im interregionalen Vergleich, Baden-Baden, 168-190.

Kailitz, Steffen, 2008a: Ein Unterschied wie Tag und Nacht? Fraktionsgeschlossenheit in Parlamentarismus und Präsidentialismus, in: ZPol 18, 291-324. 
Kailitz, Steffen, 2008b: Zwei Seiten der gleichen Medaille? Zum theoretischen und empirischen Zusammenhang zwischen der Regierungsform und der Ausgestaltung von Zweikammersystemen, in: ZParl 39, 387-414.

Kaltefleiter, Werner, 1970: Die Funktionen des Staatsoberhauptes in der parlamentarischen Demokratie, Köln/Opladen.

Kirschke, Linda, 2007: Semipresidentialism and the Perils of Power-Sharing in Neopatrimonial States, in: Comparative Political Studies 40, 1372-1394.

Kluge, Susann, 1999: Empirisch begründete Typenbildung. Zur Konstruktion von Typen und Typologien in der qualitativen Sozialforschung, Opladen.

Kluge, Susann, 2000: Empirisch begründete Typenbildung in der qualitativen Sozialforschung, http://www.qualitative-research.net/index.php/fqs/article/ view/1124/2497 (Stand: 10.12.09).

Lauth, Hans-Joachim, 2004: Demokratie und Demokratiemessung. Eine konzeptionelle Grundlegung für den interkulturellen Vergleich, Wiesbaden.

Lauth, Hans-Joachim, 2009: Typologien in der vergleichenden Politikwissenschaft: Überlegungen zum Korrespondenzproblem, in: Susanne Pickel/Gert Pickel/Hans-Joachim Lauth/Detlef Jahn (Hrsg.), Methoden der vergleichenden Politik- und Sozialwissenschaft. Neue Entwicklungen und Anwendungen, Wiesbaden, 153-172.

Lauth, Hans-Joachim/Pickel, Gert/Pickel, Susanne, 2009: Methoden der vergleichenden Politikwissenschaft. Eine Einführung, Wiesbaden.

Lazarsfeld, Paul F./Barton, Allen H., 1965: Qualitative Measurement in the Social Sciences: Classification, Typologies, and Indices, in: Daniel Lerner/ Harold D. Lasswell (Hrsg.), The Policy Sciences, Stanford, 155-192.

Lijphart, Arend, 1999: Patterns of Democracy. Government Forms and Performance in Thirty-Six Countries, Yale.

Loewenstein, Karl, 1959: Verfassungslehre, Tübingen.

McKinney, John C., 1966: Constructive Typology and Social Theory, New York.

Moestrup, Anna Sophia, 2004: Semipresidentialism in Comparative Perspective. Its Effects on Democratic Survival, Washington.

Nogueira Alcalá, Humberto, 1986a: ¿Un régimen semipresidencial para Chile?, Santiago de Chile.

Nogueira Alcalá, Humberto, 1986b: El Regimen Semipresidencial. ¿Una Nueva Forma de Gobierno Democrático?, Santiago de Chile.

Patzelt, Werner J., 2006: Parlamentsauflösung im internationalen Vergleich, in: Zeitschrift für Staats- und Europawissenschaften 4 (1), 120-141.

Protsyk, Oleh, 2005: Prime Ministers' Identity in Semi-presidential Regimes. Constitutional Norms and Cabinet Formation Outcomes, in: European Journal of Political Research 44, 721-749. 
Rogers, Rolf E., 1969: Max Weber's Ideal Type Theory, New York.

Sartori, Giovanni, 1970: Concept Misformation in Comparative Politics, in: American Political Science Review 64, 1033-1053.

Sartori, Giovanni, 1987: The Theory of Democracy Revisited, Chatham.

Schleiter, Petra/Morgan-Jones, Edward, 2009: Review Article: Citizens, Presidents and Assemblies: The Study of Semi-Presidentialism beyond Duverger and Linz, in: British Journal of Political Science 39, 871-892.

Shugart, Matthew Søberg, 1993: Of Presidents and Parliaments, in: East European Constitutional Review 2, 30-32.

Shugart, Matthew Søberg, 2005: Semi-Presidential Systems: Dual Executive and Mixed Authority Patterns, in: French Politics 3, 323-351.

Shugart, Matthew Søberg/Carey, John M., 1992: Presidents and Assemblies: Constitutional Design and Electoral Dynamics, Cambridge.

Siaroff, Alan, 2003: Comparative Presidencies: The Inadequacy of the Presidential, Semi-presidential and Parliamentary Distinction, in: European Journal of Political Research 42, 287-312.

Sodeur, Wolfgang, 1974: Empirische Verfahren zur Klassifikation, Stuttgart.

Sokal, Robert R./Sneath, Peter H., 1963: Principles of Numerical Taxonomy, San Francisco.

Steffani, Winfried, 1962: Gewaltenteilung im demokratisch-pluralistischen Rechtsstaat, in: PVS 3, 256-282.

Steffani, Winfried, 1979a: Parlamentarische und präsidentielle Demokratie. Strukturelle Aspekte westlicher Demokratien, Opladen.

Steffani, Winfried, 1979b: Strukturtypen präsidentieller und parlamentarischer Regierungssysteme, in: Winfried Steffani (Hrsg.), Parlamentarische und präsidentielle Demokratie. Strukturelle Aspekte westlicher Demokratien, Opladen, 37-60.

Steffani, Winfried, 1983: Unterscheidung parlamentarischer und präsidentieller Regierungssysteme, in: ZParl 14, 390-401.

Steffani, Winfried, 1992: Art. Parlamentarisches und präsidentielles Regierungssystem, in: Manfred G. Schmidt (Hrsg.), Die westlichen Länder, München, 288-295.

Steffani, Winfried, 1995: Semi-Präsidentialismus: ein eigenständiger Systemtyp? Zur Unterscheidung von Legislative und Parlament, in: ZParl 26, 621-641.

Steffani, Winfried, 1996: Parlamentarisch-präsidentielle „Mischsysteme“? Bemerkungen zum Stand der Forschung in der Politikwissenschaft, in: Otto Luchterhandt (Hrsg.), Neue Regierungssysteme in Osteuropa und der GUS. Probleme der Ausbildung stabiler Machtinstitutionen, Berlin, 11-62. 
Tsai, Jung-Hsiang, 2008: Sub-types of Semi-presidentialism and Political Deadlock Export, in: French Politics 61, 63-84.

Weber, Max, 1984: Soziologische Grundbegriffe, Tübingen.

Winch, Robert F., 1947: Heuristic and Empirical Typologies: a Job for Factor Analysis, in: American Sociological Review 12, 68-75.

Korrespondenzanschrift:

PD Dr. habil. Steffen Kailitz

Hannah-Arendt-Institut für Totalitarismusforschung

Helmholtzstraße 6

01069 Dresden

E-Mail: kailitz@hait.tu-dresden.de

Web: http://www.hait.tu-dresden.de/ext/ma.asp?eing=kai

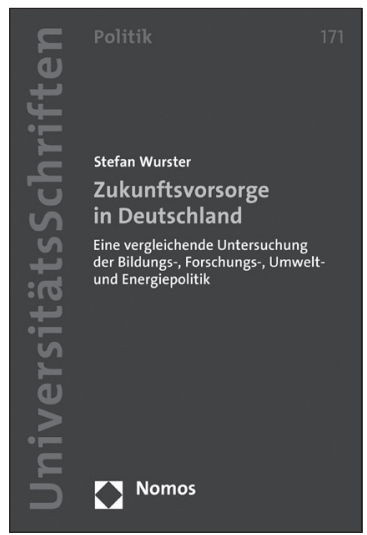

\section{Zukunftsvorsorge in Deutschland}

Eine vergleichende Untersuchung

der Bildungs-, Forschungs-, Umwelt- und

Energiepolitik

Von Stefan Wurster

2010, 432 S., brosch., 64,- $€$,

ISBN 978-3-8329-5526-7

(Nomos Universitätsschriften - Politik, Bd. 171)

Der Band geht der Frage nach, welche Leistungsperformanz die BRD in wichtigen, zukunftsvorsorgerelevanten Politikfeldern aufweist. Aus vergleichender Perspektive werden dabei politikwissenschaftliche Erklärungsfaktoren ausfindig gemacht, welche die Ursachen für das disparate Abschneiden der Bundesrepublik ausleuchten.

Bitte bestellen Sie im Buchhandel oder

versandkostenfrei unter $>$ www.nomos-shop.de

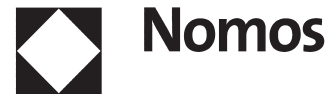

